\title{
Article \\ Polymyxin Induces Significant Transcriptomic Perturbations of Cellular Signalling Networks in Human Lung Epithelial Cells
}

\author{
Mengyao Li ${ }^{1, t, \ddagger}$, Mohammad A. K. Azad ${ }^{1, \ddagger}$, Maizbha U. Ahmed ${ }^{2}{ }^{\oplus}$, Yan Zhu ${ }^{1}{ }^{(}$, Jiangning Song $^{3,4}{ }^{\oplus}$, \\ Fanfan Zhou ${ }^{5}$, Hak-Kim Chan ${ }^{6}\left(\mathbb{D}\right.$, Tony Velkov ${ }^{7}$, Qi Tony Zhou ${ }^{2}$ and Jian $\mathrm{Li}^{1, *}$
}

check for updates

Citation: Li, M.; Azad, M.A.K.; Ahmed, M.U.; Zhu, Y.; Song, J.; Zhou, F.; Chan, H.-K.; Velkov, T.; Zhou, Q.T.; Li, J. Polymyxin Induces Significant Transcriptomic Perturbations of Cellular Signalling Networks in Human Lung Epithelial Cells. Antibiotics 2022, 11, 307. https:// doi.org/10.3390/antibiotics11030307

Academic Editor: Francesco Imperi

Received: 27 January 2022

Accepted: 21 February 2022

Published: 24 February 2022

Publisher's Note: MDPI stays neutral with regard to jurisdictional claims in published maps and institutional affiliations.

Copyright: () 2022 by the authors Licensee MDPI, Basel, Switzerland. This article is an open access article distributed under the terms and conditions of the Creative Commons Attribution (CC BY) license (https:// creativecommons.org/licenses/by/ $4.0 /)$.
1 Biomedicine Discovery Institute, Infection \& Immunity Program and Department of Microbiology, Monash University, Melbourne, VIC 3800, Australia; mengyaoli369@163.com (M.L.); mohammad.azad@monash.edu (M.A.K.A.); yan.zhu@monash.edu (Y.Z.)

2 Department of Industrial and Physical Pharmacy, College of Pharmacy, Purdue University, West Lafayette, IN 47907, USA; ahmed156@purdue.edu (M.U.A.); tonyzhou@purdue.edu (Q.T.Z.)

3 Biomedicine Discovery Institute and Department of Biochemistry and Molecular Biology, Monash University, Melbourne, VIC 3800, Australia; jiangning.song@monash.edu

4 Monash Centre for Data Science, Monash University, Melbourne, VIC 3800, Australia

5 Sydney Pharmacy School, Faculty of Medicine and Health, The University of Sydney, Sydney, NSW 2006, Australia; fanfan.zhou@sydney.edu.au

6 Advanced Drug Delivery Group, Sydney Pharmacy School, The University of Sydney, Sydney, NSW 2006, Australia; kim.chan@sydney.edu.au

7 Department of Pharmacology and Therapeutic Sciences, University of Melbourne, Melbourne, VIC 3010, Australia; tony.velkov@unimelb.edu.au

* Correspondence: jian.li@monash.edu; Tel.: +61-3-9903-9702; Fax: +61-3-9905-6450

+ Current address: Department of Critical Care Medicine, The Second Clinical Medical College, Jinan University (Shenzhen People's Hospital), Shenzhen 518020, China; Integrated Chinese and Western Medicine Postdoctoral Research Station, Jinan University, Guangzhou 510632, China.

$\ddagger$ The authors contributed equally to this work.

Abstract: Inhaled polymyxins are increasingly used to treat pulmonary infections caused by multidrugresistant Gram-negative pathogens. We have previously shown that apoptotic pathways, autophagy and oxidative stress are involved in polymyxin-induced toxicity in human lung epithelial cells. In the present study, we employed human lung epithelial cells A549 treated with polymyxin B as a model to elucidate the complex interplay of multiple signalling networks underpinning cellular responses to polymyxin toxicity. Polymyxin B induced toxicity $(1.0 \mathrm{mM}, 24 \mathrm{~h})$ in A549 cells was assessed by flow cytometry and transcriptomics was performed using microarray. Polymyxin B induced cell death was $19.0 \pm 4.2 \%$ at $24 \mathrm{~h}$. Differentially expressed genes (DEGs) between the control and polymyxin B treated cells were identified with Student's $t$-test. Pathway analysis was conducted with KEGG and Reactome and key hub genes related to polymyxin B induced toxicity were examined using the STRING database. In total we identified 899 DEGs (FDR < 0.01), KEGG and Reactome pathway analyses revealed significantly up-regulated genes related to cell cycle, DNA repair and DNA replication. NF- $\mathrm{KB}$ and nucleotide-binding oligomerization domain-like receptor (NOD) signalling pathways were identified as markedly down-regulated genes. Network analysis revealed the top 5 hub genes (i.e., degree) affected by polymyxin B treatment were PLK1 (48), CDK20 (46), CCNA2 (42), BUB1 (40) and BUB1B (37). Overall, perturbations of cell cycle, DNA damage and pro-inflammatory NF- $\mathrm{kB}$ and NOD-like receptor signalling pathways play key roles in polymyxininduced toxicity in human lung epithelial cells. Noting that NOD-like receptor signalling represents a group of key sensors for microorganisms and damage in the lung, understanding the mechanism of polymyxin-induced pulmonary toxicity will facilitate the optimisation of polymyxin inhalation therapy in patients.

Keywords: polymyxin; pulmonary toxicity; transcriptomics; lung epithelial cells 


\section{Introduction}

Antimicrobial resistance is one of the top significant threats to global public health [1]. Particularly, antibiotic resistant lower respiratory tract infections are a major cause of mortality for all ages and the leading cause of death for children under 5 years [2]. In 2016 lower respiratory tract infections caused approximately 2.38 million deaths worldwide, with over 1 million deaths occurring in adults $>70$ years old [3]. Increasing multi-drug resistance (MDR) among Gram-negative lung pathogens (e.g., Acinetobacter baumannii) is a particularly grave threat in critically ill patients such as those with ventilator-associated pneumonia (VAP) [4]. The polymyxins (polymyxin B and colistin) retain significant activity against many of these MDR pathogens [5-7], and are often the last-line therapy for the treatment of these problematic lung infections [8,9].

There have been increasing reports of polymyxin resistance in hospitals worldwide [5,10,11] Polymyxins are usually administered intravenously for treatment of infections caused by Gram-negative bacteria [12-14]. However, the current dosing recommendations for intravenous polymyxins are suboptimal, particularly for the treatment of lung infections where relatively low drug exposure is achieved in epithelial lining fluid (ELF) [14-17]. Dose-dependent nephrotoxicity that can occur in up to $60 \%$ of patients following intravenous administration of the polymyxins [18,19], is the major limiting factor for dose escalation. In contrast, inhaled polymyxins can achieve high drug exposure directly at the site of infection within the lungs while minimising systemic drug exposure [20,21]. Consequently, the use of inhaled polymyxins to treat lung infections has rapidly increased over the last decade [22,23]. However, current inhaled therapy of polymyxins is empirical and pulmonary adverse effects have been frequently reported [23-25]. Our previous studies suggest that polymyxin toxicity in human epithelial cells is due to multiple cell death pathways [26,27], the mechanisms underpinning polymyxin-induced pulmonary toxicity remains unclear and no information is available on the interplay of different signalling pathways at the systems level. Given such information is critical for the optimisation of polymyxin inhalation therapy, this study employed transcriptomics to examine polymyxininduced perturbations of signalling networks in human lung epithelial A549 cells. Our novel findings revealed that polymyxin treatment of human lung epithelial cells at concentrations achievable in the lungs via inhalation therapy induced perturbations in the expression of genes involved in cell cycle, DNA damage and pro-inflammatory NF- $\mathrm{B}$ and NOD-like receptor signalling pathways.

\section{Material and Methods}

\subsection{Cell Culture and Treatment}

For toxicity assessment, human lung alveolar epithelial A549 cells (American Type Culture Collection, Manassas, MD, USA) were grown and maintained according to the manufacturer instruction [26]. A459 cells (1.2 × $10^{5}$ cells/well) were seeded in 12-well plates (Corning Costar, Sigma-Aldrich, Australia) and incubated at $37^{\circ} \mathrm{C}$ in a humidified atmosphere containing $5 \% \mathrm{CO}_{2}$ for $24 \mathrm{~h}$ prior to experiments. At $24 \mathrm{~h}$, the medium was removed, and fresh medium was added for treatments. Immediately prior to all experiments, solutions of polymyxin B (Beta Pharma, Shanghai, China) were prepared in Milli-Q water (Millipore, North Ryde, Australia) and sterilised using $0.20-\mu \mathrm{m}$ cellulose acetate syringe filters (Millipore, Bedford, MA, USA). A549 cells were exposed to either $1.0 \mathrm{mM}$ of polymyxin B (treatment group) or Milli-Q water (control group) for $24 \mathrm{~h}(n=3)$. Polymyxin B toxicity was measured using Annexin-V-Alexa fluor 488 and propidium iodide (PI) staining with fluorescence activated cell sorting (FACS) [26].

\subsection{Gene Expression Profiling}

For the transcriptomic study, A549 cells were grown in 6-well plates $\left(1 \times 10^{6}\right.$ cells / well $)$ overnight. Cells were exposed for $24 \mathrm{~h}$ to either polymyxin B (1.0 mM) or Milli-Q water (control) $(n=3)$. Subsequently, the growth medium was aspirated, and lysis buffer was added to the cell-culture plates. A rubber policeman was used to detach the cells. The lysate 
was passed through a blunt 20-gauge needle fitted to an RNase-free syringe at least 5 times and RNA was extracted using a RNeasy Plus Mini Kit (QIAGEN, Australia). Quantification of extracted mRNA was performed by the Monash Health Translation Precinct (MHTP) Medical Genomic Facility with Agilent microarray using the Human Gene Expression v3 [28].

\subsection{Bioinformatic Analysis}

Microarray raw intensities were pre-processed to perform background correction, quantile normalisation and $\log _{2}$ transformation by Bioconductor package Limma [29]. In cases of multiple probes being mapped to the same gene, the expression value was summarised as the arithmetic mean of the values of the multiple probes (on the $\log _{2}$ scale). After data preprocessing, 21,755 genes were obtained for analysis. Differentially expressed genes (DEGs) were identified using Student's $t$-test with a false discovery rate $($ FDR $)<0.01$. All $p$ values were adjusted by the Benjamini-Hochberg FDR procedure [30]. The key genes affected in polymyxin B-treated cells were selected by partial least squaresdiscriminant analysis (PLS-DA). Functional pathway analysis was conducted against the Kyoto Encyclopedia of Genes and Genomes (KEGG) [31] and Reactome hierarchy [32]. The hypergeometric distribution was used to determine whether a pathway was over-presented in the interesting gene list (i.e. DEG list).

The protein-protein interaction (PPI) network was generated with the STRING database [33]. Genes were represented as "nodes", while the interactions between any two genes/proteins were represented as an "edge". In the STRING database, interactions between target genes and their functional partners in the network were determined by confidence scores [33]. To ensure binary interactions with highest confidence were generated, interactions with confidence scores $\geq 0.95$ were selected. After filtration, 134,240 relationships between 9380 human proteins were collected and networks were visualized by Cytoscape [34].

\section{Results}

Flow cytometry analysis revealed that following exposure to $1.0 \mathrm{mM}$ of polymyxin $\mathrm{B}$ for $24 \mathrm{~h}, 80.9 \pm 4.1 \%$ of A549 cells were alive and the percentages of early apoptotic, late apoptotic and necrotic cells were low. Therefore, this treatment condition (1.0 $\mathrm{mM}$ for $24 \mathrm{~h})$ was applied for the subsequent transcriptomics study. In total, 899 DEGs were identified in response to polymyxin B treatment, comprising 514 up-regulated and 385 down-regulated genes (FDR $\leq 0.01)$. The top 15 genes affected by polymyxin B were identified using variable influence on projection (VIP) scores (Figure 1). Nine genes (CD86, ALDOC, MT1F, PCSK9, FGFBP1, MVD, INSIG1, RAB33A and CYB5B) were up-regulated and six downregulated (BEX2, CCL5, FAM129A, CSTA, NUPR1 and TXNIP) in polymyxin B-treated cells. The CD86 gene, which encodes a potent co-stimulator of $\mathrm{T}$ and $\mathrm{B}$ lymphocyte function, had the highest VIP score (11.8) (Figure 1). The identified DEGs were comprised by cellular processes including metabolic pathways (CYB5B, INSIG1, MVD and ALDOC), immune function (CD86, CCL5, TXNIP and ALDOC), gene expression (TXNIP), and signal transduction (CD86, FGFBP1 and CCL5).

\subsection{Cell Cycle, DNA Replication and DNA Repair Were Up-Regulated by Polymyxin B Treatment}

KEGG pathway enrichment analysis showed that the 514 genes up-regulated by polymyxin B treatment were significantly (FDR $<0.05$ ) associated with steroid biosynthesis, cell cycle, the Fanconi anemia pathway, DNA replication, mismatch repair, homologous recombination, terpenoid backbone biosynthesis, mineral absorption, oocyte meiosis and fatty acid biosynthesis (Table 1). Furthermore, Reactome analysis revealed that these genes were significantly enriched in pathways related to cell cycle $\left(100\right.$, FDR $\left.=4.8 \times 10^{-14}\right)$, DNA replication $\left(24, \mathrm{FDR}=2.2 \times 10^{-6}\right)$, DNA repair $\left(39, \mathrm{FDR}=2.9 \times 10^{-5}\right)$, and reproduction $\left(18, \mathrm{FDR}=3.4 \times 10^{-4}\right)$. Cell cycle, M phase, cell cycle checkpoints, mitotic prometaphase and chromatin maintenance were affected by polymyxin treatment (Table S1). Cell cycle was significantly enriched by up-regulated genes in both the KEGG and Reactome databases. Notably, all four phases of the cell cycle, namely G1, S, G2 and M phases were perturbed 
(Figure 2). Six DEGs (RPA1, H2AFX, HIST1H2BJ, HIST1H2BE, HIST1H2BB and HIST4H4) were shared by five cell cycle-related pathways in Reactome, including the cell cycle, cell cycle checkpoints, chromosome maintenance, meiosis, and cell cycle mitotic pathways (Figure 3).

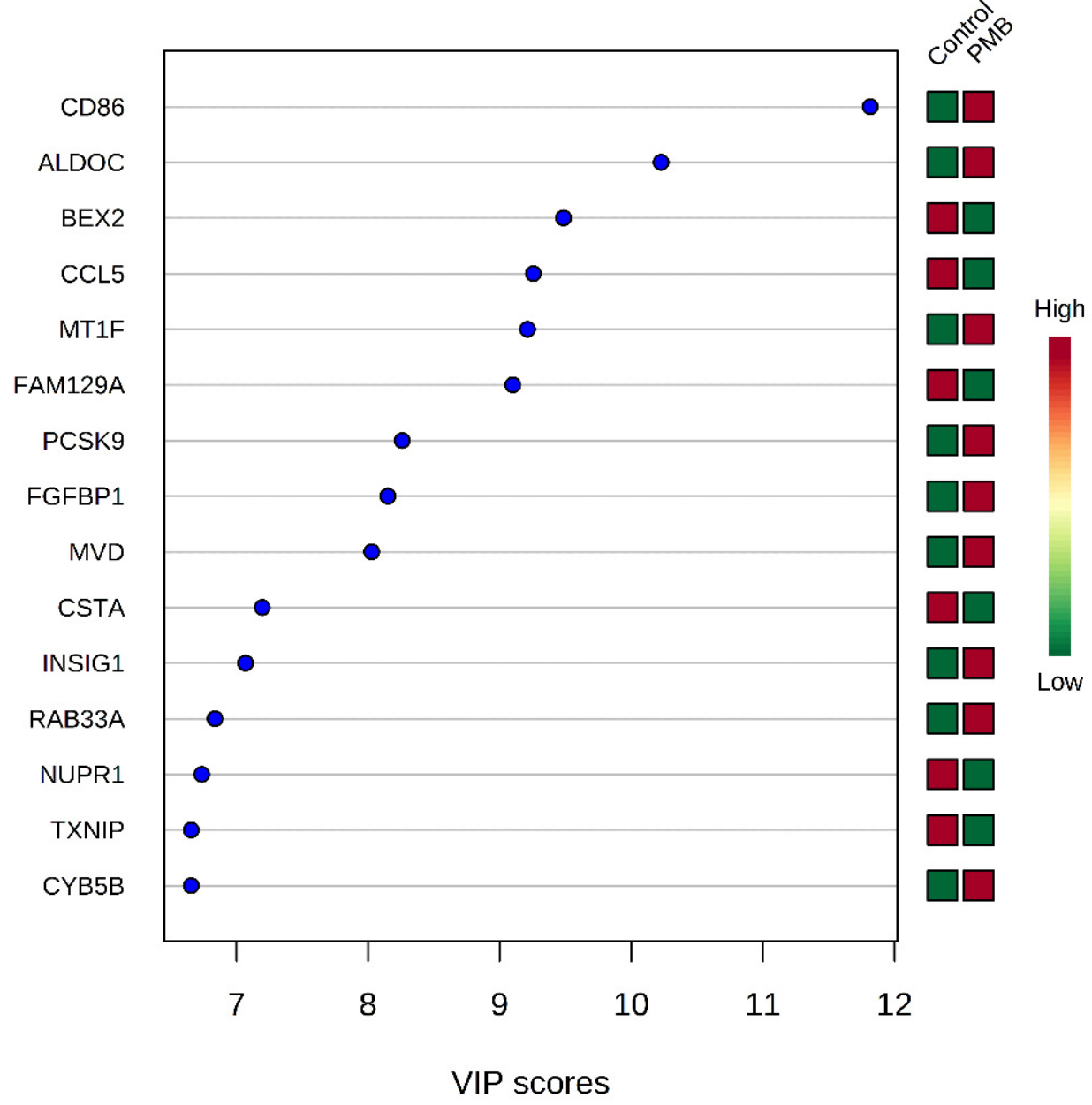

Figure 1. The top 15 genes affected by polymyxin B treatment as identified using variable influence on projection (VIP) scores. Key genes were identified using partial least squares-discriminant analysis (PLS-DA). VIP score and heatmap indicate the relative expression level of the corresponding gene in each group. $\mathrm{PMB}$, polymyxin $\mathrm{B}$.

Table 1. Polymyxin B up-regulated the expression of 514 genes in A549 cells enriched by KEGG pathway analysis (FDR < 0.05). k: number of DEGs in a pathway; m: number of background genes in a pathway; FDR: adjusted hypergeometric $p$ value.

\begin{tabular}{ccccc}
\hline KEGG ID & Pathway & k & m & FDR \\
\hline hsa00100 & Steroid biosynthesis & 12 & 19 & $8.1 \times 10^{-12}$ \\
hsa04110 & Cell cycle & 23 & 124 & $5.2 \times 10^{-10}$ \\
hsa03460 & Fanconi anaemia pathway & 13 & 52 & $4.1 \times 10^{-7}$ \\
hsa03030 & DNA replication & 9 & 36 & $7.7 \times 10^{-5}$ \\
hsa03430 & Mismatch repair & 7 & 23 & $0.2 \times 10^{-3}$ \\
hsa03440 & Homologous recombination & 9 & 41 & $0.2 \times 10^{-3}$ \\
hsa00900 & Terpenoid backbone biosynthesis & 6 & 22 & $0.2 \times 10^{-2}$ \\
hsa04978 & Mineral absorption & 8 & 50 & $0.5 \times 10^{-2}$ \\
hsa04114 & Oocyte meiosis & 12 & 119 & 0.01 \\
hsa00061 & Fatty acid biosynthesis & 4 & 13 & 0.01 \\
\hline
\end{tabular}




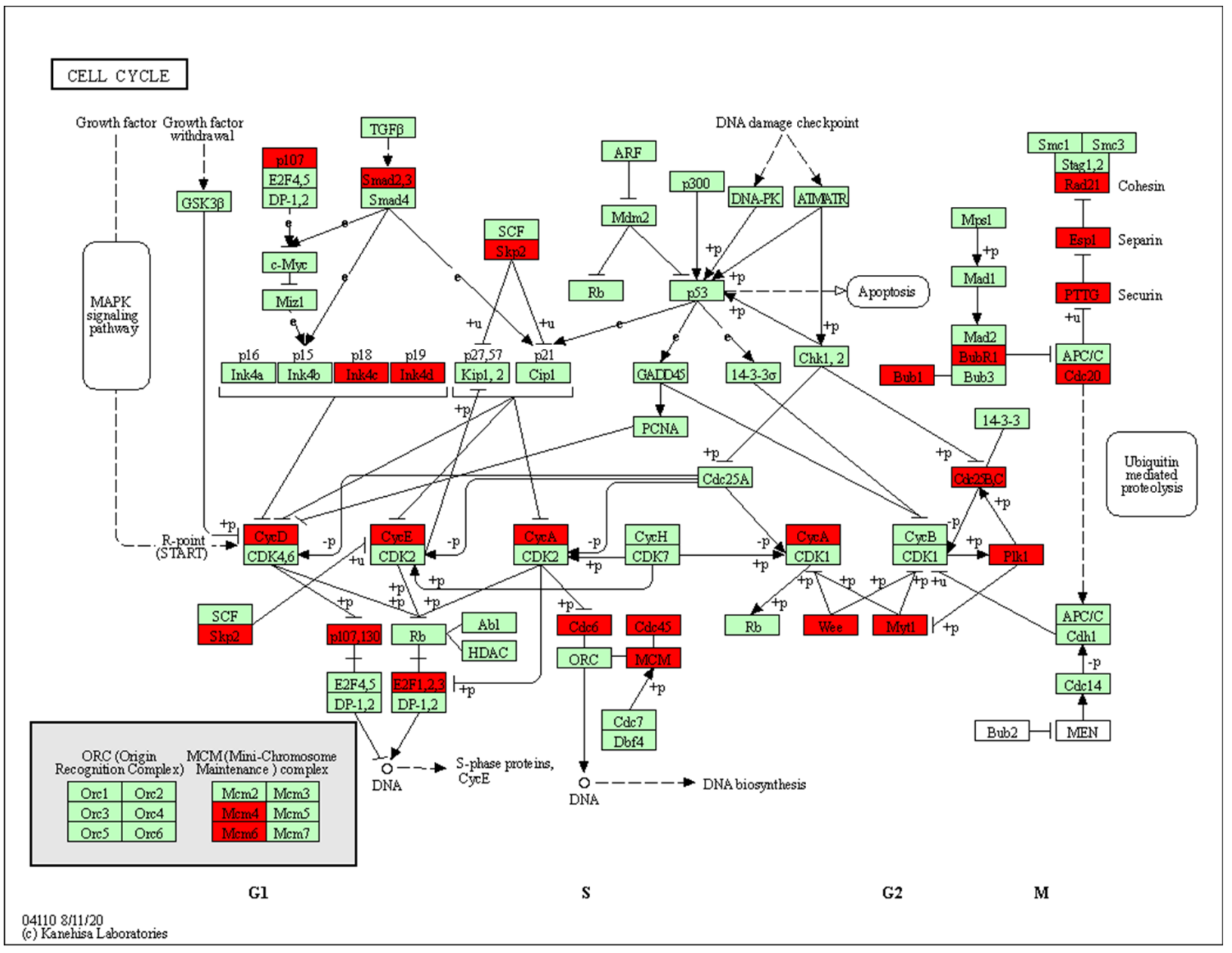

Figure 2. Cell cycle perturbations by polymyxin B. Red boxes present the proteins encoded by up-regulated genes; green boxes present the proteins encoded by down-regulated genes.

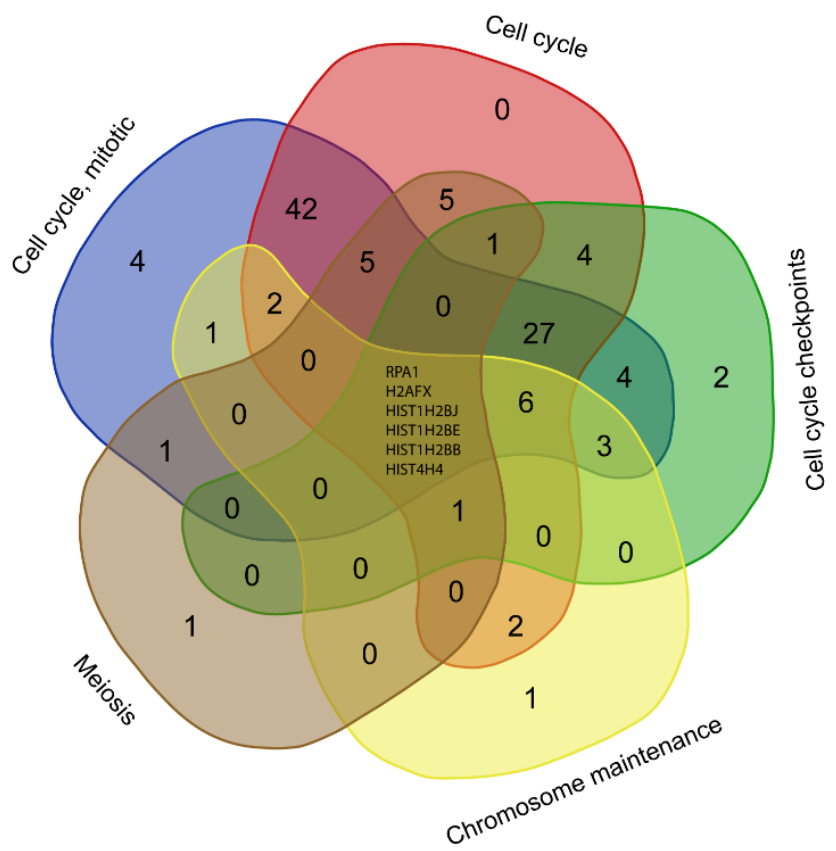

Figure 3. Common DEGs involved in the cell cycle pathways due to polymyxin B treatment. 
DNA replication and repair were also significantly perturbed across 23 up-regulated genes (Tables 1 and S1). Nine up-regulated genes involved in DNA replication were identified using both KEGG and Reactome, including helicase encoding gene DNA2, ligase encoding gene LIG1, minichromosome maintenance complex components MCM4 and MCM6, DNA polymerases POLA2 and POLD3, replication factors RFC2 and RFC5, and replication protein RPA1. In the significantly enriched KEGG pathways, the Fanconi anemia, mismatch repair and homologous recombination pathways were associated with DNA repair. By mapping DEGs to these three pathways in KEGG and to DNA repair pathways in Reactome, 19 shared genes were identified (Figure 4). The mineral absorption pathway in KEGG was significantly enriched by 8 up-regulated DEGs, comprising 6 metallothioneinencoding genes (MT1A, MT1B, MT1E, MT1F, MT1H and MT1X), the sulphate/anion transporter gene $S L C 26 A 9$, and the zinc transporter gene ZNT1 (Table 1).

\section{A. DNA replication}

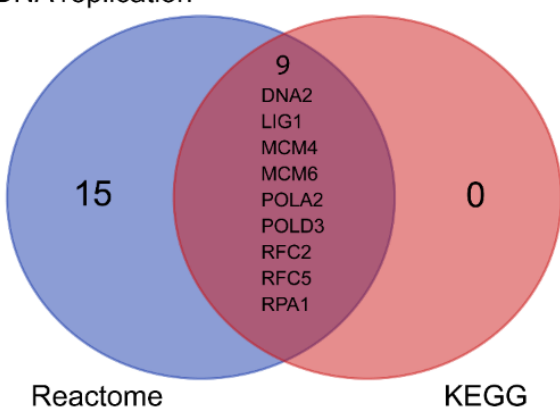

B. DNA repair

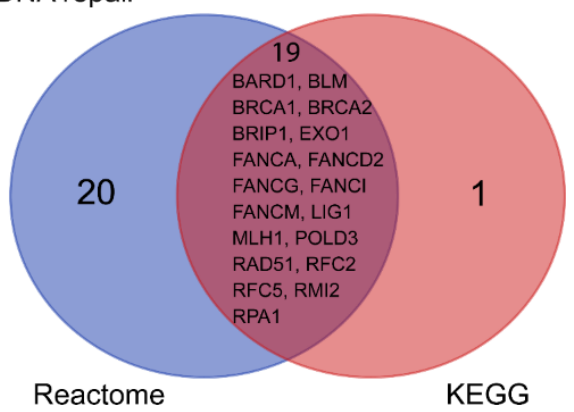

Figure 4. Common up-regulated genes in response to polymyxin treatment shared by Reactome and KEGG. (A) Shared up-regulated DEGs in the DNA replication pathway; (B) shared up-regulated DEGs in the DNA repair pathway.

\subsection{NF- $\mathrm{BB}$ and NOD-Like Receptor Signalling Pathways Were Down-Regulated by Polymyxin $B$ Treatment}

Analysis with KEGG and Reactome with FDR $<0.05$ did not reveal any pathways enriched by the 385 genes down-regulated in response to polymyxin B treatment. However, when the threshold of $p$ value $<0.05$ was applied, several pathways were significantly enriched in both KEGG and Reactome. In KEGG, aminoacyl-tRNA biosynthesis, amino acid metabolism, NOD-like receptor signalling, apoptosis and NF- $\kappa$ B signalling pathways were affected by polymyxin B treatment (Table 2). In Reactome, pathways responsible for signal transduction were perturbed, including TGF-beta receptor signalling activates SMADs, calcitonin-like ligand receptors, regulation of TNFR1 signalling, NOD1/2 signalling pathway, TNFR1-induced NF- $\mathrm{kB}$ signalling pathway, and TNF signalling (Table S2). Shared by both enrichment methods, NF- $\kappa B$ and NOD-like receptor signalling were the common perturbed pathways and BIRC3, TNFAIP3 and TRAF1 were the common DEGs in the NF- $\mathrm{BB}$ signalling. Four down-regulated genes, namely BIRC3, TNFAIP3, CASP4 and RIPK2 were all mapped to NOD-like receptor signalling. 
Table 2. Polymyxin B down-regulated the expression of 385 genes in A549 cells enriched by KEGG pathway analysis $(p<0.05)$. $\mathrm{k}$ : number of DEGs in a pathway; $\mathrm{m}$ : number of background genes in a pathway; $p: p$ value.

\begin{tabular}{ccccc}
\hline KEGG ID & Pathway & k & m & $p$ \\
\hline hsa00970 & Aminoacyl-tRNA biosynthesis & 6 & 44 & $0.3 \times 10^{-3}$ \\
hsa00260 & Glycine, serine and threonine metabolism & 5 & 39 & $0.1 \times 10^{-2}$ \\
hsa00290 & Valine, leucine and isoleucine biosynthesis & 2 & 4 & $0.3 \times 10^{-2}$ \\
hsa04621 & NOD-like receptor signalling pathway & 10 & 170 & $0.3 \times 10^{-2}$ \\
hsa04210 & Apoptosis & 8 & 135 & $0.8 \times 10^{-2}$ \\
hsa00270 & Cysteine and methionine metabolism & 4 & 46 & 0.02 \\
hsa04064 & NF-kB signalling pathway & 5 & 90 & 0.04 \\
\hline
\end{tabular}

\subsection{Key Regulatory Genes of the Cell Cycle Perturbed by Polymyxin B}

Using protein-protein interaction (PPI) network analysis, 134,240 relationships between 9380 human proteins were collected with the confidence score of $\geq 0.95$. Next, the DEGs induced by polymyxin B treatment were overlaid. The largest connected component was composed of 142 nodes and 804 edges (Figure 5A). STRING network analysis revealed that the top 5 regulatory hub genes with the highest degrees in the network due to polymyxin B treatment were PLK1 (48), CDC20 (46), CCNA2 (42), BUB1 (40) and BUB1B (37) (Table 3). DEGs in Component A were mostly involved with cell cycle. The death receptor signalling component (Figure $5 \mathrm{~B}$ ) consisted of five down-regulated genes, including BIRC3 (3) and TRAF1 (2); DEGs in Component $C$ (Figure 5C) were responsible for cholesterol biosynthesis, with CYP51A1 (11) having the highest degree in this component. Component $\mathrm{D}$ was associated with plasma lipoprotein clearance and signalling by the TGF-beta receptor complex, and Component $\mathrm{E}$ with mRNA splicing. DEGs from the smaller components (Figure 5F-I) were associated with GPCR ligand binding, glycolysis, intraflagellar transport and fatty acyl-CoA biosynthesis. The GPCR ligand binding component was constructed by two up-regulated genes (HTR7 and ADM) and three down-regulated genes (CALCA, ADM2 and PTHLH) (Figure 5F). The glycolysis component (Figure 5G) also contained two up-regulated (PGAM1 and ENO2) and three down-regulated genes (PSAT1, $B P G M$ and $P H G D H$ ). The fatty acyl-CoA biosynthesis component (Figure 5I) was comprised of four up-regulated genes, namely ACACA (2), FASN (2), ACSS2 (1) and ACSL3 (1).

Table 3. Top 10 perturbed hub genes identified by protein-protein interaction network.

\begin{tabular}{lccc}
\hline Node & Name & Direction & Degree \\
\hline PLK1 & Polo-like kinase 1 & $\mathrm{Up}$ & 48 \\
CDC20 & Cell division cycle 20 homolog & $\mathrm{Up}$ & 46 \\
CCNA2 & Cyclin A2 & $\mathrm{Up}$ & 42 \\
BUB1 & Budding uninhibited by benzimidazoles 1 homolog & $\mathrm{Up}$ & 40 \\
BUB1B & Budding uninhibited by benzimidazoles 1 homolog beta & $\mathrm{Up}$ & 37 \\
AURKB & Aurora kinase B & $\mathrm{Up}$ & 36 \\
NDC80 & NDC80 homolog, kinetochore complex component & $\mathrm{Up}$ & 35 \\
KIF11 & Kinesin family member 11 & 35 \\
CENPE & Centromere protein E, 312kDa & 33 \\
CDCA8 & Cell division cycle associated 8 & $\mathrm{Up}$ & 33 \\
\hline
\end{tabular}


- Up-regulated

- Down-regulated

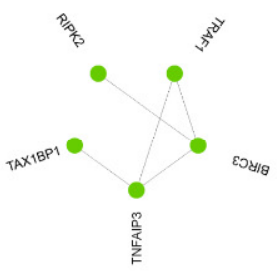

B. Death receptor signalling

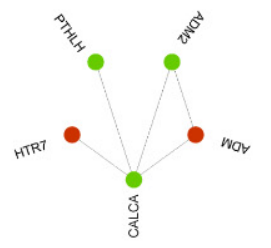

F. GPCR ligand binding

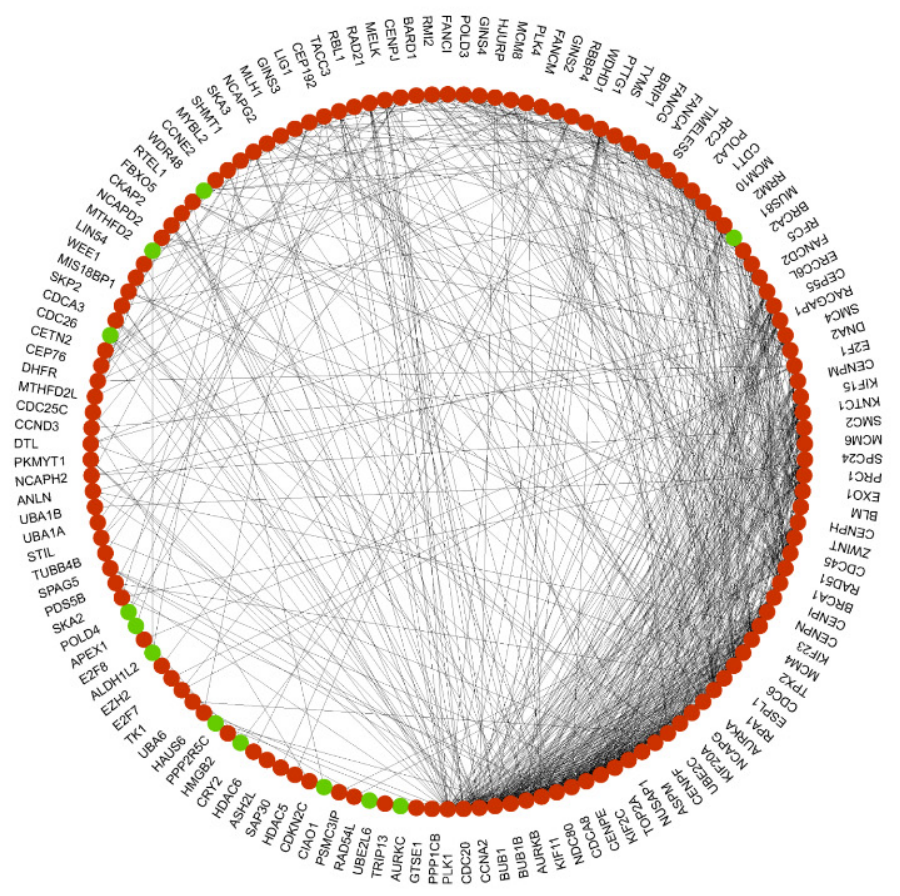

A. Cell cycle
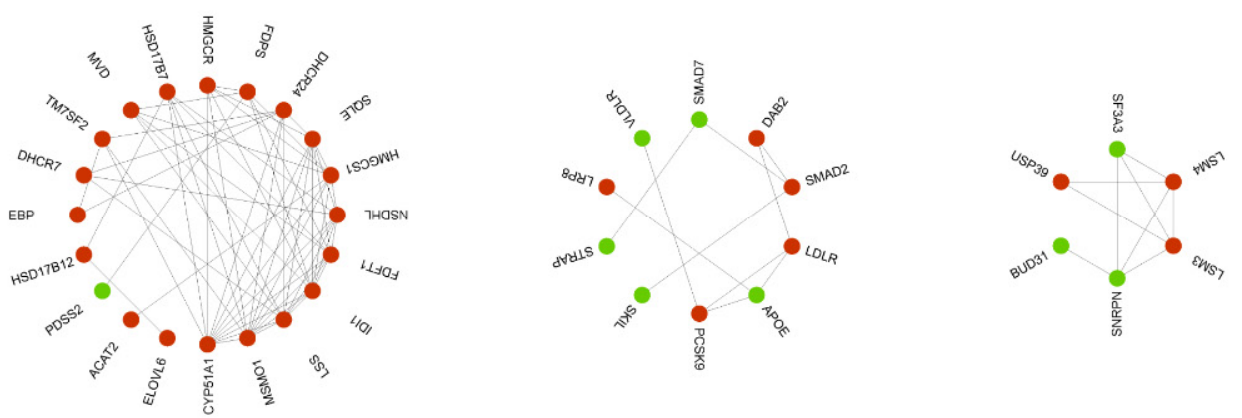

C. Cholesterol biosynthesis
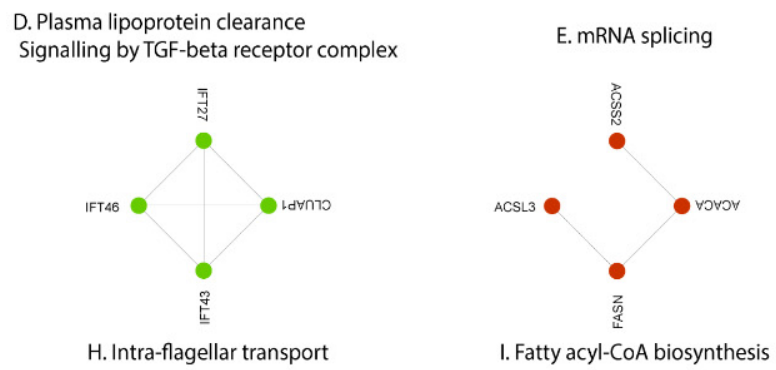

Figure 5. Perturbations of the signalling networks in A549 cells induced by polymyxin B treatment. (A) Cell cycle is the largest connected component containing 142 nodes and 804 edges; (B) Death receptor signalling component is consisted of 5 down-regulated genes; (C) Cholesterol biosynthesis component contains 19 up-regulated genes and 1 down-regulated genes; (D) Component D shows 5 up-regulated and 5 down-regulated genes which are related to plasma lipoprotein clearance and signalling by TGF-beta receptor complex; (E) mRNA splicing component contains 3 up-regulated and 3 down-regulated genes; both (F) GPCR ligand binding and (G) Glycolysis components are comprised of 2 up-regulated and 3 down-regulated genes; $(\mathbf{H})$ Intra-flagellar transport component contains 4 down-regulated genes, while (I) Fatty acyl-COA biosynthesis component contains 4 up-regulated genes. Red and green nodes are up-regulated and down-regulated genes, respectively, following polymyxin B treatment. Interactions between proteins are indicated by lines. 


\section{Discussion}

A major limitation of intravenous polymyxin administration to patients with lung infections is very low drug exposure in the ELF [15-17]. The aerosolised direct delivery of polymyxins to the lungs via a nebuliser or as a dry powder circumvents this limitation and as such have a prominent use for the treatment of VAP and cystic fibrosis associated lung infections [35]. Inhalation of polymyxins provides substantial pharmacokinetic/pharmacodynamic (PK/PD) advantages by achieving significantly higher lung exposure than is possible with intravenous administration, while simultaneously minimising systemic exposure and thus nephrotoxicity $[17,20]$. Indeed, inhaled polymyxins as salvage therapy for the treatment of respiratory tract infections caused by MDR Gramnegative pathogens have shown excellent bacterial killing and high rates of clinical cure and improvement in patients with nosocomial pneumonia [17,36,37].

Despite the potential advantages of aerosolised polymyxins, pulmonary toxicity has nevertheless been reported in patients $[26,36]$. Our group has previously shown that polymyxin B can cause the activation of caspase-3, 8, and 9, increased expression of the cell surface death receptor FasL, and mitochondrial damage in human lung epithelial A549 cells in a dose- and time-dependent manner [26]. Moreover, the subcellular localisation of polymyxin B in mitochondria, early endosomes and lysosomes of A549 cells indicates the critical role of cellular uptake in its respiratory toxicity [27]. Given that polymyxinassociated toxicities are multifaceted, in the present study we investigated how polymyxin B-induced gene expression profiles in human A549 lung epithelial cells contribute to lung injury. The concentration of polymyxin $B(1.0 \mathrm{mM})$ was chosen considering clinical relevance and resulted in the death of $\sim 19 \%$ of A549 cells following $24 \mathrm{~h}$ of treatment (Figure S1). This degree of toxicity is in agreement with earlier studies where mild toxicity in A549 cells was observed following similar polymyxin B exposure $[26,27]$. This sub-lethal concentration of polymyxin $B$ also avoided the activation of non-specific cellular processes in necrotic cells that could degrade mRNA and thus ensured the availability of a sufficient number of viable cells for collection of quality mRNA for analysis [38]. Such information will be critical for the safe delivery of polymyxins to the lungs. The correlative analyses of DEGs, functional enrichment and PPI network revealed not only the key genes which mediated polymyxin-induced cell death, but also regulation of the signalling pathways to trigger cell death. This finding is well-aligned with the substantial increase in late apoptotic cell death following treatment of polymyxin B in the present study (Figure S1).

A major finding of the present study is that polymyxin treatment significantly perturbed cell cycle, DNA repair, NF-kB and NOD-like receptor signalling pathways in A549 cells. Our correlative bioinformatic analysis revealed that several pathways differentially enriched by polymyxin B treatment were involved in the regulation of cellular processes for cell survival. In particular, pathways involved with cellular proliferation, apoptosis and inflammation were enriched, including cell cycle, DNA replication, mismatch repair, and signalling pathways (e.g., NF-KB and NOD-like receptor signalling). Similar enrichment of these pathways was observed following pathway analysis using the Reactome database. We recently reported in human renal proximal tubular HK-2 cells DNA damage and cell cycle arrest were induced by polymyxins [39]. Taken together, our results strongly indicate that DNA damage and cell cycle play key roles in polymyxin-induced toxicity in A549 cells. Further molecular biology and in vivo studies are warranted to confirm these findings.

The Fanconi anaemia pathway repairs DNA inter-strand crosslinks that inhibit DNA replication and transcription [40]. Enrichment of the Fanconi anaemic pathway in the current study indicates a protective response against DNA damage caused by a sublethal dose of polymyxin $\mathrm{B}$. Histones are the major protein components involved in the compaction of genomic DNA into higher order structures, with histones $\mathrm{H} 2 \mathrm{~A}$ with $\mathrm{H} 2 \mathrm{~B}, \mathrm{H} 3$, H4 and H1 (linker) constituting the nucleosome core [41]. Once chromatin is compacted, genes become inaccessible to transcription factors, preventing their expression [42]. The progression of cells from the G1 to S phase, that latter being the phase in which DNA is replicated, increase the rate of histone gene transcription up to 5-fold and results in a 
35-fold increase in the histone level [43]. Once the $S$ phase is completed, histone mRNA levels rapidly decrease [43]. We observed significant up-regulation of the histone cluster 1 , 2, 4 gene family (HIST1H1C, HIST1H2BB, HIST1H2AL, HIST1H2BE, HIST1H3D, HIST1H3E, HIST1H3G, HIST1H2BJ, HIST2H3D and HIST4H4) in polymyxin B treated A549 cells; these results indicate that polymyxin $B$ caused arrest of the cell cycle in the $S$ phase, possibly due to cells inability to repair the polymyxin B induced DNA damage. Furthermore, PPI network analysis revealed key DEGs in cell cycle due to polymyxin B treatment (Figure 5A) and by ranking with their degrees the top five DEGs in the largest component include PLK1, CDC20, CCNA2, BUB1 and BUB1B. PLK1 encodes a Ser/Thr protein kinase which belongs to the CDC5/Polo subfamily and is highly expressed during mitosis [44]. PLK1 is also a key regulator of mitosis initiation via control of the CDK1/Cyclin B complex activity which drives the transition of cells from G2 into the M phase [45]. CDC20 encodes celldivision cycle protein 20, an essential regulator of cell division which activates the anaphase promoting complex $(\mathrm{APC} / \mathrm{C}$ ) responsible for the ubiquitination and degradation of securin and cyclin B ubiquitination, thus promoting the onset of anaphase and mitotic exit [46]. Immunostaining and confocal microscopy studies previously revealed the ubiquitin protein and polymyxin B co-localised in A549 cells after treatment with $0.1 \mathrm{mM}$ polymyxin B for $24 \mathrm{~h}$ [27]. CCNA2 encodes cyclin A2, a core cell cycle regulator that participates in controlling both $S$ phase and mitosis by binding to CDK kinases [47]. Multidomain protein kinases BUB1 and BUB1B are the central components of the mitotic checkpoint for spindle assembly [48]. The PPI network analysis results further confirmed that polymyxin B disturbed cell cycle by affecting the expression levels of multiple key regulatory genes.

Polymyxin B also perturbed key regulators of several other important cellular functions, including death receptor signalling, cholesterol biosynthesis, glycolysis and fatty acyl-CoA biosynthesis (Figure 5B,C,G,I). BIRC3, which was down-regulated by polymyxin $\mathrm{B}$ in the present study (Figure $5 \mathrm{~B}$ ), is involved in the inhibition of apoptosis by binding to tumour necrosis factor receptor-associated factors TRAF1/2 [49]. CYP51A1 encodes a cytochrome P450 monooxygenase involved in sterol biosynthesis that catalyses the removal of a 14 alpha-methyl group from lanosterol [50]; whereas MSMO1 encodes methylsterol monooxygenase 1 which catalyses the first step in the removal of the two C-4 methyl groups of 4,4-dimethylzymosterol (Figure 5C) [51]. Cholesterol plays an important role in lung immunity and host reprogramming of cholesterol metabolism is critical to defending pathogens $[52,53]$. Cholesterol enhances host defense signalling by promoting the assembly and activation of multiple receptors, including Toll-like receptors (TLRs), $\mathrm{T}$ cell receptors, B cell receptors and MHCII [54]. Phosphoglycerate mutase 1 (PGAM1) catalyses the interconversion of 3-phosphoglycerate and 2-phosphoglycerate in glycolysis (Figure 5G) [55]. During lung injury, hypoxia-inducible factors (HIFs) can transcriptionally induce the expression of multiple genes encoding key enzymes of glycolysis in alveolar epithelial cells [56]. Significantly increased expression of cholesterol biosynthesis and glycolysis suggests that host immunity was activated by polymyxin B. The acyl-CoA synthetase short chain family member 2 (EC:6.2.1.1) encoded by ACSS2 participates in fatty acyl-CoA biosynthesis by mediating acetyl-CoA synthesis from acetate (Figure 5I) [57]. Although these pathways (Figure 5B-I) were not considered enriched in the KEGG or Reactome analyses, PPI network analysis indicated their perturbation via key regulatory genes by polymyxin $B$ treatment.

NF- $\mathrm{KB}$ and NOD-like receptor signalling was significantly enriched by down-regulated genes due to polymyxin B treatment (Table 2). NOD-like receptor is a pattern recognition receptor involved in the recognition of bacterial pathogens in the lungs and chronic sterile inflammatory diseases, such as pneumonia, acute lung injury, chronic obstructive pulmonary disease, pneumoconiosis, acute respiratory distress syndrome and asthma [58]. The best characterised NODs are NOD1 and NOD2; and their stimulation can result in the activation of MAPK and NF- $\mathrm{BB}$ [59]. Activated NF- $\mathrm{B}$ leads to the up-regulated transcription and production of inflammatory mediators [59]. Although NF- $\kappa$ B and NOD-like receptor signalling pathways were enriched from down-regulated genes by polymyxin $B$ 
treatment, several of these genes play a suppressive role in the pathways. cIAP2 (encoded by BIRC3) acts as a ubiquitin-protein ligase, negatively regulating NF- $\mathrm{B}$ and NOD-like receptor signalling pathway $[60,61]$. TNFAIP3 is a terminator of NF- $\kappa B$ signalling, which is a central regulator of immunopathology [62]. Therefore, down-regulation of these negative regulators may suggest potential activation of NF- $\mathrm{BB}$ and NOD-like receptor signalling pathways. Perturbations of these pro-inflammatory pathways indicate that polymyxin B treatment might trigger immune responses in human lung epithelial A549 cells; and highlights that a drug-host-pathogen interplay most likely underlies polymyxin therapy in patients with lung infections.

Although CD86 (FDR $=0.03, \mathrm{FC}=1.7)$ was not identified as a DEG by a cut-off of FDR $<0.01$, it is the gene with the largest fold change in the polymyxin B-treated cells (Figure 1). CD86 encodes a potent co-stimulator of T and B lymphocyte function [63] and our result indicates that polymyxin B has immune modulatory activity in A549 cells (Figure 1). Activation of $\mathrm{T}$ cells requires the interaction of the T-cell receptor with an antigen bound to MHC expressed on antigen-presenting cells, as well as a costimulatory signal [64]. Increased surface expression of the costimulatory molecule CD86 has been reported in monocyte-derived dendritic cells exposed to several other cationic antimicrobial peptides [65]. The over expression of CD86 in polymyxin B-treated A549 cells suggests the potential role of polymyxins in modulating both innate and acquired immunity by the activation of costimulatory molecules.

Another major finding of the present study is that six metallothionein encoding genes (MT1A, MT1B, MT1E, MT1F, MT1H and MT1X) were significantly up-regulated in response to polymyxin B treatment (Table 1). Metallothioneins are a family of low molecular weight, cysteine-rich proteins that strongly bind and exchange specific metal ions, in particular zinc $[66,67]$. Metallothioneins plays an important role in homeostasis of essential metals (e.g., zinc and copper), detoxification of toxic metals (e.g., cadmium), and protection against oxidative stress $[68,69]$. Zinc, cadmium, oxidative stress and/or inflammation can rapidly and substantially up-regulate the transcription of metallothionein genes [70]. Interestingly, a recent study showed significant correlation between the accumulation of polymyxin and increased concentration of intracellular zinc in A549 cells [71]. It has also been demonstrated that polymyxins cause oxidative stress in A549 cells [26]. These results well support the up-regulated metallothionein encoding genes observed in the present study (Figure S2). As stress proteins, metallothioneins might be a hallmark of polymyxin-induced lung toxicity, which requires further investigation.

In conclusion, this study is the first correlative analysis of transcriptomics, pathways and PPI network of human lung epithelial A549 cells in response to polymyxin treatment. Our findings demonstrate a complex interaction of signalling networks related to DNA damage, DNA repair and cell cycle in A549 cells in response to polymyxin B treatment. This study provides key systems pharmacological information for optimizing pulmonary delivery of polymyxins in patients and the ongoing development of safer, new-generation polymyxins.

Supplementary Materials: The following are available online at https:/ /www.mdpi.com/article/10 .3390/antibiotics11030307/s1, Figure S1: A549 cell viability as measured by flow cytometry, Figure S2: Activation of the antioxidant network system in A549 cells following the treatment with polymyxin B. Genes in red were up-regulated. Table S1: Reactome pathways enriched by up-regulated DEGs, Table S2: Reactome pathways enriched by down-regulated DEGs.

Author Contributions: M.L., M.A.K.A. and J.L. conceived the study; M.A.K.A. and M.U.A. conducted the experiments; M.L. and Y.Z. were responsible for the data analysis; J.S., F.Z. and H.-K.C. reviewed and edited the manuscript; T.V., Q.T.Z. and J.L. acquired the funding. All authors have read and agreed to the published version of the manuscript. 
Funding: This study was funded by the Australian National Health and Medical Research Council (NHMRC, APP1104581) and the National Institute of Allergy and Infectious Diseases of the National Institutes of Health (T.Q.Z., J.L. and T.V., NIH R01 AI132681 and R01 AI146160). The content is solely the responsibility of the authors and does not necessarily represent the official views of the National Institute of Allergy and Infectious Diseases or the National Institutes of Health. J.L. is an NHMRC Principal Research Fellow and T.V. is an NHMRC Career Development Industrial Fellow.

Institutional Review Board Statement: Not applicable.

Informed Consent Statement: Not applicable.

Data Availability Statement: The microarray data are available from the authors upon reasonable request.

Conflicts of Interest: The authors declare no conflict of interest.

Transparency Declarations: Jian Li and Tony Velkov are listed as inventors on the patent application WO2015149131 'Polymyxin Derivatives as Antimicrobial Compounds' which has been licenced to Qpex Biopharma.

\section{References}

1. World Health Organization. Ten Threats to Global Health in 2019. Available online: https://www.who.int/news-room/spotlight/ ten-threats-to-global-health-in-2019 (accessed on 20 February 2022).

2. Naghavi, M.; Abajobir, A.A.; Abbafati, C.; Abbas, K.M.; Abd-Allah, F.; Abera, S.F.; Aboyans, V.; Adetokunboh, O.; Afshin, A.; Agrawal, A.; et al. Global, regional, and national age-sex specific mortality for 264 causes of death, 1980-2016: A systematic analysis for the Global Burden of Disease Study 2016. Lancet 2017, 390, 1151-1210. [CrossRef]

3. Troeger, C.; Blacker, B.; Khalil, I.A.; Rao, P.C.; Cao, J.; Zimsen, S.R.M.; Albertson, S.B.; Deshpande, A.; Farag, T.; Abebe, Z.; et al. Estimates of the global, regional, and national morbidity, mortality, and aetiologies of lower respiratory infections in 195 countries, 1990-2016: A systematic analysis for the Global Burden of Disease Study 2016. Lancet Infect. Dis. 2018, 18, 1191-1210. [CrossRef]

4. Gupta, R.; Malik, A.; Rizvi, M.; Ahmed, M.; Singh, A. Epidemiology of multidrug-resistant Gram-negative pathogens isolated from ventilator-associated pneumonia in ICU patients. J. Glob. Antimicrob. Resist. 2017, 9, 47-50. [CrossRef] [PubMed]

5. Castanheira, M.; Deshpande, L.M.; Mendes, R.E.; Canton, R.; Sader, H.S.; Jones, R.N. Variations in the occurrence of resistance phenotypes and carbapenemase genes among Enterobacteriaceae isolates in 20 years of the SENTRY Antimicrobial Surveillance Program. Open Forum Infect. Dis. 2019, 6 (Suppl. 1), S23-S33. [CrossRef]

6. China Antimicrobial Surveillance Network. 2020. Available online: http:// chinets.com/Data/AntibioticDrugFast (accessed on 20 February 2022).

7. Walkty, A.; DeCorby, M.; Nichol, K.; Karlowsky, J.A.; Hoban, D.J.; Zhanel, G.G. In vitro activity of colistin (polymyxin E) against 3,480 isolates of Gram-negative bacilli obtained from patients in Canadian hospitals in the CANWARD study, 2007-2008. Antimicrob. Agents Chemother. 2009, 53, 4924-4926. [CrossRef]

8. Theuretzbacher, U.; Gottwalt, S.; Beyer, P.; Butler, M.; Czaplewski, L.; Lienhardt, C.; Moja, L.; Paul, M.; Paulin, S.; Rex, J.H.; et al Analysis of the clinical antibacterial and antituberculosis pipeline. Lancet Infect. Dis. 2019, 19, e40-e50. [CrossRef]

9. Theuretzbacher, U.; Outterson, K.; Engel, A.; Karlen, A. The global preclinical antibacterial pipeline. Nat. Rev. Microbiol. 2020, 18, 275-285. [CrossRef]

10. Li, Z.; Cao, Y.; Yi, L.; Liu, J.H.; Yang, Q. Emergent polymyxin resistance: End of an era? Open Forum Infect. Dis. 2019, 6, ofz368 [CrossRef]

11. Yu, Y.; Andrey, D.O.; Yang, R.S.; Sands, K.; Tansawai, U.; Li, M.; Portal, E.; Gales, A.C.; Niumsup, P.R.; Sun, J.; et al. A Klebsiella pneumoniae strain co-harbouring mcr-1 and mcr-3 from a human in Thailand. J. Antimicrob. Chemother. 2020, 75, 2372-2374. [CrossRef]

12. Nation, R.L.; Li, J.; Cars, O.; Couet, W.; Dudley, M.N.; Kaye, K.S.; Mouton, J.W.; Paterson, D.L.; Tam, V.H.; Theuretzbacher, U.; et al. Framework for optimisation of the clinical use of colistin and polymyxin B: The Prato polymyxin consensus. Lancet Infect. Dis. 2015, 15, 225-234. [CrossRef]

13. Nation, R.L.; Garonzik, S.M.; Thamlikitkul, V.; Giamarellos-Bourboulis, E.J.; Forrest, A.; Paterson, D.L.; Li, J.; Silveira, F.P. Dosing guidance for intravenous colistin in critically-ill patients. Clin. Infect. Dis. 2017, 64, 565-571. [CrossRef] [PubMed]

14. Nang, S.C.; Azad, M.A.K.; Velkov, T.; Zhou, Q.T.; Li, J. Rescuing the last-line polymyxins: Achievements and challenges. Pharmacol. Rev. 2021, 73, 679-728. [CrossRef] [PubMed]

15. Garonzik, S.M.; Li, J.; Thamlikitkul, V.; Paterson, D.L.; Shoham, S.; Jacob, J.; Silveira, F.P.; Forrest, A.; Nation, R.L. Population pharmacokinetics of colistin methanesulfonate and formed colistin in critically ill patients from a multicenter study provide dosing suggestions for various categories of patients. Antimicrob. Agents Chemother. 2011, 55, 3284-3294. [CrossRef]

16. Sandri, A.M.; Landersdorfer, C.B.; Jacob, J.; Boniatti, M.M.; Dalarosa, M.G.; Falci, D.R.; Behle, T.F.; Bordinhao, R.C.; Wang, J.; Forrest, A.; et al. Population pharmacokinetics of intravenous polymyxin B in critically ill patients: Implications for selection of dosage regimens. Clin. Infect. Dis. 2013, 57, 524-531. [CrossRef] [PubMed] 
17. Yapa, S.W.S.; Li, J.; Patel, K.; Wilson, J.W.; Dooley, M.J.; George, J.; Clark, D.; Poole, S.; Williams, E.; Porter, C.J.; et al. Pulmonary and systemic pharmacokinetics of inhaled and intravenous colistin methanesulfonate in cystic fibrosis patients: Targeting advantage of inhalational administration. Antimicrob. Agents Chemother. 2014, 58, 2570-2579. [CrossRef] [PubMed]

18. Pogue, J.M.; Lee, J.; Marchaim, D.; Yee, V.; Zhao, J.J.; Chopra, T.; Lephart, P.; Kaye, K.S. Incidence of and risk factors for colistin-associated nephrotoxicity in a large academic health system. Clin. Infect. Dis. 2011, 53, 879-884. [CrossRef]

19. Kubin, C.J.; Ellman, T.M.; Phadke, V.; Haynes, L.J.; Calfee, D.P.; Yin, M.T. Incidence and predictors of acute kidney injury associated with intravenous polymyxin B therapy. J. Infect. 2012, 65, 80-87. [CrossRef]

20. Boisson, M.; Jacobs, M.; Gregoire, N.; Gobin, P.; Marchand, S.; Couet, W.; Mimoz, O. Comparison of intrapulmonary and systemic pharmacokinetics of colistin methanesulfonate (CMS) and colistin after aerosol delivery and intravenous administration of CMS in critically ill patients. Antimicrob. Agents Chemother. 2014, 58, 7331-7339. [CrossRef]

21. Naesens, R.; Vlieghe, E.; Verbrugghe, W.; Jorens, P.; Ieven, M. A retrospective observational study on the efficacy of colistin by inhalation as compared to parenteral administration for the treatment of nosocomial pneumonia associated with multidrugresistant Pseudomonas aeruginosa. BMC Infect. Dis. 2011, 11, 317. [CrossRef]

22. Michalopoulos, A.; Papadakis, E. Inhaled anti-infective agents: Emphasis on colistin. Infection 2010, 38, 81-88.

23. Velkov, T.; Abdul Rahim, N.; Zhou, Q.T.; Chan, H.K.; Li, J. Inhaled anti-infective chemotherapy for respiratory tract infections: Successes, challenges and the road ahead. Adv. Drug Deliv. Rev. 2015, 85, 65-82. [CrossRef] [PubMed]

24. Lin, Y.W.; Zhou, Q.; Onufrak, N.J.; Wirth, V.; Chen, K.; Wang, J.; Forrest, A.; Chan, H.K.; Li, J. Aerosolized polymyxin B for treatment of respiratory tract infections: Determination of pharmacokinetic-pharmacodynamic indices for aerosolized polymyxin B against Pseudomonas aeruginosa in a mouse lung infection model. Antimicrob. Agents Chemother. 2017, 61, e00211-17. [CrossRef] [PubMed]

25. Lin, Y.W.; Zhou, Q.T.; Cheah, S.E.; Zhao, J.; Chen, K.; Wang, J.; Chan, H.K.; Li, J. Pharmacokinetics/Pharmacodynamics of pulmonary delivery of colistin against Pseudomonas aeruginosa in a mouse lung infection model. Antimicrob. Agents Chemother. 2017, 61, e02025-16. [CrossRef] [PubMed]

26. Ahmed, M.U.; Velkov, T.; Lin, Y.W.; Yun, B.; Nowell, C.J.; Zhou, F.; Zhou, Q.T.; Chan, K.; Azad, M.A.K.; Li, J. Potential toxicity of polymyxins in human lung epithelial cells. Antimicrob. Agents Chemother. 2017, 61, e02690-16. [CrossRef] [PubMed]

27. Ahmed, M.U.; Velkov, T.; Zhou, Q.T.; Fulcher, A.J.; Callaghan, J.; Zhou, F.; Chan, K.; Azad, M.A.K.; Li, J. Intracellular localization of polymyxins in human alveolar epithelial cells. J. Antimicrob. Chemother. 2019, 74, 48-57. [CrossRef]

28. Tran, T.B.; Zhu, Y.; Johnson, M.D.; Kaye, K.S.; Bergen, P. Systems pharmacology of interactions among Acinetobacter baumannii, human lung epithelial cells and polymyxin B. In Proceedings of the European Congress of Clinical Microbiology \& Infectious Diseases (ECCMID), Vienna, Austria, 22-25 April 2017.

29. Yang, Y.H.; Dudoit, S.; Luu, P.; Lin, D.M.; Peng, V.; Ngai, J.; Speed, T.P. Normalization for cDNA microarray data: A robust composite method addressing single and multiple slide systematic variation. Nucleic Acids Res. 2002, 30, e15. [CrossRef]

30. Smyth, G.K.; Speed, T. Normalization of cDNA microarray data. Methods 2003, 31, 265-273. [CrossRef]

31. Benjamini, Y.; Hochberg, Y. Controlling the false discovery rate: A practical and powerful approach to multiple testing. J. R. Stat. Soc. Ser. B (Methodological) 1995, 57, 289-300. [CrossRef]

32. Gillespie, M.; Jassal, B.; Stephan, R.; Milacic, M.; Rothfels, K.; Senff-Ribeiro, A.; Griss, J.; Sevilla, C.; Matthews, L.; Gong, C.; et al The reactome pathway knowledgebase 2022. Nucleic Acids Res. 2022, 50, D687-D692. [CrossRef]

33. Szklarczyk, D.; Gable, A.L.; Lyon, D.; Junge, A.; Wyder, S.; Huerta-Cepas, J.; Simonovic, M.; Doncheva, N.T.; Morris, J.H.; Bork, P.; et al. STRING v11: Protein-protein association networks with increased coverage, supporting functional discovery in genome-wide experimental datasets. Nucleic Acids Res. 2019, 47, D607-D613. [CrossRef]

34. Shannon, P.; Markiel, A.; Ozier, O.; Baliga, N.S.; Wang, J.T.; Ramage, D.; Amin, N.; Schwikowski, B.; Ideker, T. Cytoscape: A software environment for integrated models of biomolecular interaction networks. Genome Res. 2003, 13, 2498-2504. [CrossRef] [PubMed]

35. Michalopoulos, A.; Fotakis, D.; Virtzili, S.; Vletsas, C.; Raftopoulou, S.; Mastora, Z.; Falagas, M.E. Aerosolized colistin as adjunctive treatment of ventilator-associated pneumonia due to multidrug-resistant Gram-negative bacteria: A prospective study. Respir Med. 2008, 102, 407-412. [CrossRef] [PubMed]

36. Pereira, G.H.; Muller, P.R.; Levin, A.S. Salvage treatment of pneumonia and initial treatment of tracheobronchitis caused by multidrug-resistant Gram-negative bacilli with inhaled polymyxin B. Diagn. Microbiol. Infect. Dis. 2007, 58, 235-240. [CrossRef] [PubMed]

37. Hansen, C.R.; Pressler, T.; Hoiby, N. Early aggressive eradication therapy for intermittent Pseudomonas aeruginosa airway colonization in cystic fibrosis patients: 15 years experience. J. Cyst. Fibros. 2008, 7, 523-530. [CrossRef] [PubMed]

38. Kukurba, K.R.; Montgomery, S.B. RNA Sequencing and Analysis. Cold Spring Harb. Protoc. 2015, 2015, pdb-top084970. [CrossRef]

39. Yun, B.; Zhang, T.; Azad, M.A.K.; Wang, J.; Nowell, C.J.; Kalitsis, P.; Velkov, T.; Hudson, D.F.; Li, J. Polymyxin B causes DNA damage in HK-2 cells and mice. Arch. Toxicol. 2018, 92, 2259-2271. [CrossRef]

40. Ceccaldi, R.; Sarangi, P.; D'Andrea, A.D. The Fanconi anaemia pathway: New players and new functions. Nat. Rev. Mol. Cell Biol. 2016, 17, 337-349. [CrossRef]

41. Kornberg, R.D.; Lorch, Y. Twenty-five years of the nucleosome, fundamental particle of the eukaryote chromosome. Cell 1999, 98, 285-294. [CrossRef] 
42. Kalashnikova, A.A.; Rogge, R.A.; Hansen, J.C. Linker histone H1 and protein-protein interactions. Biochim. Biophys. Acta 2016, 1859, 455-461. [CrossRef]

43. Marzluff, W.F.; Duronio, R.J. Histone mRNA expression: Multiple levels of cell cycle regulation and important developmental consequences. Curr. Opin. Cell Biol. 2002, 14, 692-699. [CrossRef]

44. Golsteyn, R.M.; Mundt, K.E.; Fry, A.M.; Nigg, E.A. Cell cycle regulation of the activity and subcellular localization of Plk1, a human protein kinase implicated in mitotic spindle function. J. Cell Biol. 1995, 129, 1617-1628. [CrossRef] [PubMed]

45. Gavet, O.; Pines, J. Activation of cyclin B1-Cdk1 synchronizes events in the nucleus and the cytoplasm at mitosis. J. Cell Biol. 2010, 189, 247-259. [CrossRef] [PubMed]

46. Yu, H. Cdc20: A WD40 activator for a cell cycle degradation machine. Mol. Cell 2007, 27, 3-16. [CrossRef] [PubMed]

47. Pagano, M.; Pepperkok, R.; Verde, F.; Ansorge, W.; Draetta, G. Cyclin A is required at two points in the human cell cycle. EMBO J. 1992, 11, 961-971. [CrossRef]

48. Bolanos-Garcia, V.M.; Blundell, T.L. BUB1 and BUBR1: Multifaceted kinases of the cell cycle. Trends Biochem. Sci. 2011, 36, 141-150. [CrossRef]

49. Roy, N.; Deveraux, Q.L.; Takahashi, R.; Salvesen, G.S.; Reed, J.C. The c-IAP-1 and c-IAP-2 proteins are direct inhibitors of specific caspases. EMBO J. 1997, 16, 6914-6925. [CrossRef]

50. Stromstedt, M.; Rozman, D.; Waterman, M.R. The ubiquitously expressed human CYP51 encodes lanosterol 14 alpha-demethylase, a cytochrome P450 whose expression is regulated by oxysterols. Arch. Biochem. Biophys. 1996, 329, 73-81. [CrossRef]

51. Porter, T.D. Electron transfer pathways in cholesterol synthesis. Lipids 2015, 50, 927-936. [CrossRef]

52. Fessler, M.B. The intracellular cholesterol landscape: Dynamic integrator of the immune response. Trends Immunol. 2016, 37, 819-830. [CrossRef]

53. Shaikh, S.R.; Fessler, M.B.; Gowdy, K.M. Role for phospholipid acyl chains and cholesterol in pulmonary infections and inflammation. J. Leukoc. Biol. 2016, 100, 985-997. [CrossRef]

54. Fessler, M.B.; Parks, J.S. Intracellular lipid flux and membrane microdomains as organizing principles in inflammatory cell signaling. J. Immunol. 2011, 187, 1529-1535. [CrossRef] [PubMed]

55. Fothergill-Gilmore, L.A.; Watson, H.C. The phosphoglycerate mutases. Adv. Enzymol. Relat. Areas Mol. Biol. 1989, 62, $227-313$.

56. Vohwinkel, C.U.; Hoegl, S.; Eltzschig, H.K. Hypoxia signaling during acute lung injury. J. Appl. Physiol. 2015, 119, 1157-1163. [CrossRef] [PubMed]

57. Wellen, K.E.; Hatzivassiliou, G.; Sachdeva, U.M.; Bui, T.V.; Cross, J.R.; Thompson, C.B. ATP-citrate lyase links cellular metabolism to histone acetylation. Science 2009, 324, 1076-1080. [CrossRef]

58. Balamayooran, T.; Balamayooran, G.; Jeyaseelan, S. Review: Toll-like receptors and NOD-like receptors in pulmonary antibacterial immunity. Innate Immun. 2010, 16, 201-210. [CrossRef]

59. Shaw, M.H.; Reimer, T.; Kim, Y.G.; Nunez, G. NOD-like receptors (NLRs): Bona fide intracellular microbial sensors. Curr. Opin. Immunol. 2008, 20, 377-382. [CrossRef] [PubMed]

60. Gyrd-Hansen, M.; Meier, P. IAPs: From caspase inhibitors to modulators of NF-kappaB, inflammation and cancer. Nat. Rev. Cancer 2010, 10, 561-574. [CrossRef]

61. Evans, P.C.; Ovaa, H.; Hamon, M.; Kilshaw, P.J.; Hamm, S.; Bauer, S.; Ploegh, H.L.; Smith, T.S. Zinc-finger protein A20, a regulator of inflammation and cell survival, has de-ubiquitinating activity. Biochem. J. 2004, 378, 727-734. [CrossRef]

62. Shembade, N.; Harhaj, E.W. Regulation of NF-kappaB signaling by the A20 deubiquitinase. Cell. Mol. Immunol. 2012, 9, 123-130. [CrossRef]

63. Castell, J.V.; Donato, M.T.; Gomez-Lechon, M.J. Metabolism and bioactivation of toxicants in the lung. The in vitro cellular approach. Exp. Toxicol. Pathol. 2005, 57 (Suppl. 1), 189-204. [CrossRef]

64. Gimmi, C.D.; Freeman, G.J.; Gribben, J.G.; Sugita, K.; Freedman, A.S.; Morimoto, C.; Nadler, L.M. B-cell surface antigen B7 provides a costimulatory signal that induces T cells to proliferate and secrete interleukin 2. Proc. Natl. Acad. Sci. USA 1991, 88, 6575-6579. [CrossRef] [PubMed]

65. Diamond, G.; Beckloff, N.; Weinberg, A.; Kisich, K.O. The roles of antimicrobial peptides in innate host defense. Curr. Pharm. Des. 2009, 15, 2377-2392. [CrossRef] [PubMed]

66. Hamer, D.H. Metallothionein. Annu. Rev. Biochem. 1986, 55, 913-951. [CrossRef] [PubMed]

67. Davis, S.R.; Cousins, R.J. Metallothionein expression in animals: A physiological perspective on function. J. Nutr. 2000, 130, 1085-1088. [CrossRef]

68. Kagi, J.H. Overview of metallothionein. Methods Enzymol. 1991, 205, 613-626.

69. Kang, Y.J. Metallothionein redox cycle and function. Exp. Biol. Med. 2006, 231, 1459-1467. [CrossRef]

70. Andrews, G.K. Regulation of metallothionein gene expression by oxidative stress and metal ions. Biochem. Pharmacol. 2000, 59, 95-104. [CrossRef]

71. Azad, M.A.K.; Zhang, S.; Li, J.; Kim, Y.; Yu, H.H.; Fulcher, A.J.; Howard, D.L.; de Jonge, M.D.; James, S.A.; Roberts, K.D.; et al. Synchrotron-based X-ray fluorescence microscopy reveals accumulation of polymyxins in single human alveolar epithelial cells. Antimicrob. Agents Chemother. 2021, 65, e02314-20. [CrossRef] 